\title{
Trends and Emissions of Six Perfluorocarbons in the Northern and Southern Hemisphere: Supplementary Material
}

Elise S. Droste ${ }^{1}$, Karina E. Adcock ${ }^{1}$, Matthew J. Ashfold ${ }^{2}$, Charles Chou $^{3}$, Zoë Fleming ${ }^{4, *}$, Paul J. Fraser ${ }^{5}$, Lauren J. Gooch ${ }^{1}$, Andrew J. Hind ${ }^{1}$, Ray L. Langenfelds ${ }^{5}$, Emma Leedham Elvidge ${ }^{1}$, Norfazrin Mohd Hanif ${ }^{1,6}$, Simon O’Doherty ${ }^{7}$, David E. Oram ${ }^{1,8}$, Chang-Feng Ou-Yang ${ }^{9}$, Marios Panagi ${ }^{4}$, Claire E. Reeves ${ }^{1}$, William T. Sturges ${ }^{1}$, and Johannes C. Laube ${ }^{1,10}$

${ }^{1}$ Centre for Ocean and Atmospheric Sciences, School of Environmental Sciences, University of East Anglia, Norwich, NR4 7TJ, UK

${ }^{2}$ School of Environmental and Geographical Sciences, University of Nottingham Malaysia, 43500 Semenyih, Malaysia

${ }^{3}$ Research Center for Environmental Changes, Academia Sinica, Taipei 11529, Taiwan

${ }^{4}$ National Centre for Atmospheric Science (NCAS), Department of Chemistry, University of Leicester, UK

${ }^{5}$ Commonwealth Scientific and Industrial Research Organisation, Oceans and Atmosphere, Climate Science Centre, Aspendale, Australia

${ }^{6}$ School of Environmental and Natural Resource Sciences, Faculty of Science and Technology, Universiti Kebangsaan Malaysia, 43600 Bangi, Selangor, Malaysia

${ }^{7}$ Department of Chemistry, University of Bristol, Bristol, UK

${ }^{8}$ National Centre for Atmospheric Science, School of Environmental Sciences, University of East Anglia, Norwich, NR4 7TJ, UK

${ }^{9}$ Department of Atmospheric Sciences, National Central University, Taoyuan, Taiwan

${ }^{10}$ Institute of Energy and Climate Research - Stratosphere (IEK-7), Forschungszentrum Jü lich GmbHJ, Jülich, Germany

* now at Center for Climate and Resilience Research (CR2), University of Chile, Santiago, Chile

Correspondence: E. S. Droste (e.droste@uea.ac.uk)

\section{Contents}

1 Perfluorocarbon Ratios

2 Ion Ratios

3 Uncertainties

5 Correlations of PFC Mixing Ratios in Taiwan 


\section{Perfluorocarbon Ratios}

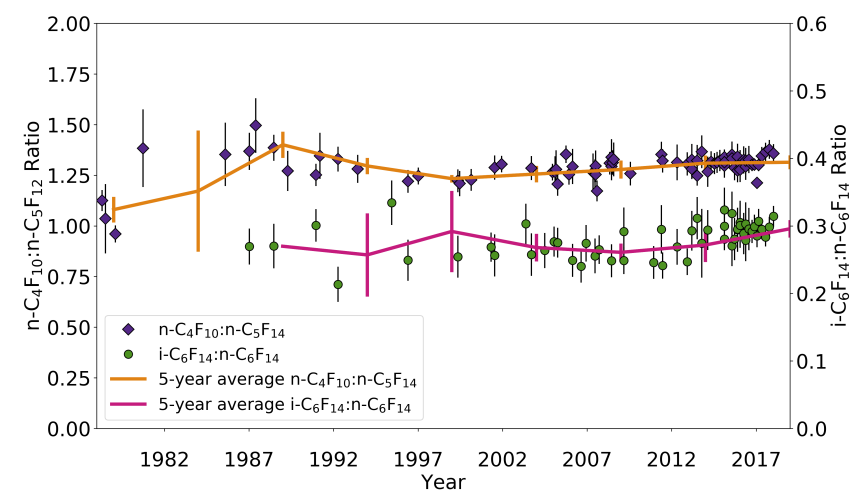

Figure S1. Ratio of $\mathrm{n}-\mathrm{C}_{4} \mathrm{~F}_{10}$ :n- $\mathrm{C}_{5} \mathrm{~F}_{12}$ (diamonds) and $\mathrm{i}-\mathrm{C}_{6} \mathrm{~F}_{14}: \mathrm{n}-\mathrm{C}_{6} \mathrm{~F}_{14}$ (circles) mixing ratios measured in Cape Grim samples between 1978 and 2018 (see Section ??). Error bars indicate propagated measurement uncertainties. Full orange and magenta lines illustrate respective five-year averages of the ratios, with error bars comprising the propagated measurement uncertainties. 


\section{Ion Ratios}

The ratio of the two main quantifying ions for $\mathrm{C}_{6} \mathrm{~F}_{14}$ and $\mathrm{C}_{7} \mathrm{~F}_{16}(\mathrm{~m} / z, 169.0$ and 219.0) is used to determine whether all isomers have been separated for a particular peak in the chromatograph. Deviation of the ratio measured in the Cape Grim samples from the ratio measured in the calibration samples, which are composed of highly purified isomers, indicates the possibility

5 that not all isomers have been separated during gas-chromatography.

For $\mathrm{i}-\mathrm{C}_{6} \mathrm{~F}_{14}$, the average ion ratio in the calibration is $0.89 \pm 0.03$ and Cape Grim air samples have an ion ratio that is on average $1.00 \pm 0.07$ (Fig. S2 A). The positive offset of the Cape Grim ion ratio to the calibration ion ratio for $\mathrm{i}-\mathrm{C}_{6} \mathrm{~F}_{14}$ seems to be consistent. $n-\mathrm{C}_{6} \mathrm{~F}_{14}$ measured in air samples have an average ion ratio of $0.99 \pm 0.03$, which is consistently - but not significantly - lower than the ion ratio for the calibrations (1.04 \pm 0.07$)$ (Fig. S2 B). Finally, $n-\mathrm{C}_{7} \mathrm{~F}_{16}$ has an average ion ratio 10 of $1.00 \pm 0.05$ in the Cape Grim air samples, which agrees very well with the average ion ratio in its calibration: $1.02 \pm 0.07$ (Fig. S2 C). Overall, the difference between the ion ratios of the air samples and the calibrations is not significant within the uncertainties, especially for $n-C_{6} F_{14}$ and $n-C_{7} F_{16}$. Even though this means that it is highly likely that only one isomer is being measured instead of multiple under one peak, the possibility that not all isomers within this peak have been separated cannot be excluded.

A limitation is that a trend analysis of the ion ratio for these PFC isomers is not possible here, since problems with baseline distortions in the Cape Grim data resulted in a lack of samples before 2005 that have good precisions on both $\mathrm{m} / \mathrm{z} 219.0$ and 169.0 ions. This is especially the case for the $m / z 219$ ion, which generally exhibits smaller peaks than the $m / z$. 169 ion and also has a noisier baseline in our analytical system. 

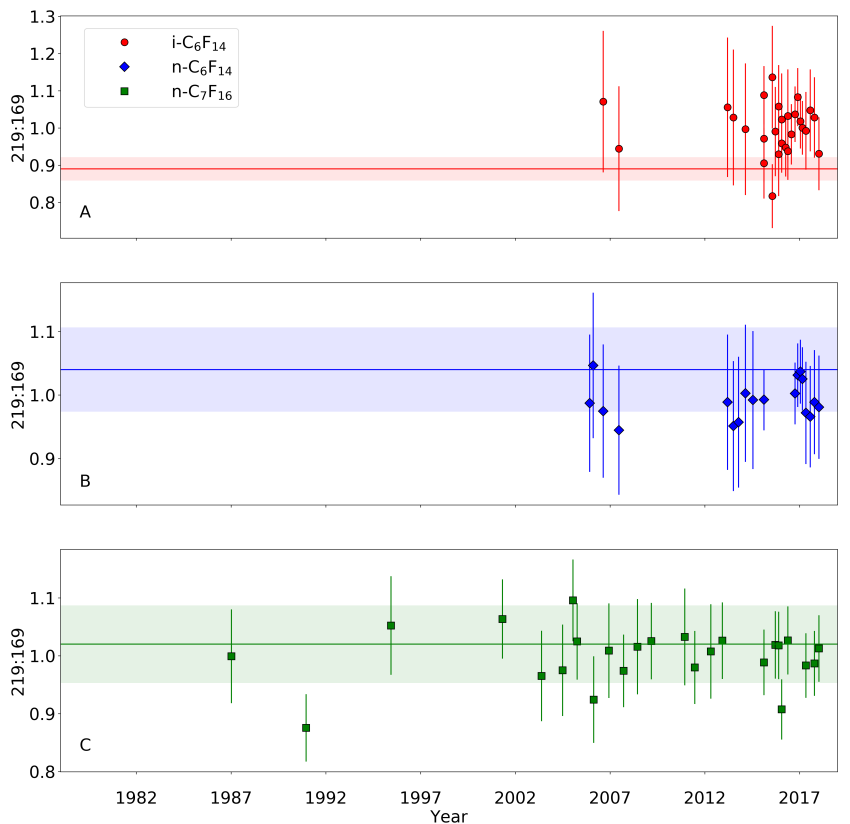

Figure S2. Ratios of ions $m / z$ 219:169 for i- $\mathrm{C}_{6} \mathrm{~F}_{14}(\mathrm{~A}), \mathrm{n}-\mathrm{C}_{6} \mathrm{~F}_{14}(\mathrm{~B})$, and $\mathrm{n}-\mathrm{C}_{7} \mathrm{~F}_{16}(\mathrm{C})$ for Cape Grim air samples between 1987 and 2018. Note that both ions could only be measured with high precision for samples after 2005 for $\mathrm{i}-\mathrm{C}_{6} \mathrm{~F}_{14}$ and $\mathrm{n}-\mathrm{C}_{6} \mathrm{~F}_{14}$. The horizontal line illustrates the average 219:169 ion ratio of the calibrations done in the current work. Shaded area indicates the propagated uncertainty of the average 219:169 ratio of the calibrations. 


\section{Uncertainties}

Table S1. Trend uncertainties (composed of the average analytical uncertainty, the average best-fit uncertainty, and the model transport uncertainty) and total uncertainties (composed as the trend uncertainty and calibration uncertainty) for all PFCs.

\begin{tabular}{ccc}
\hline $\mathrm{PFC}$ & Trend Uncertainty [\%] & Total Uncertainty [\%] \\
\hline $\mathrm{c}-\mathrm{C}_{4} \mathrm{~F}_{8}$ & 5.7 & 12.7 \\
$\mathrm{n}-\mathrm{C}_{4} \mathrm{~F}_{10}$ & 5.9 & 7.6 \\
$\mathrm{n}-\mathrm{C}_{5} \mathrm{~F}_{12}$ & 8.2 & 13.4 \\
$\mathrm{i}-\mathrm{C}_{6} \mathrm{~F}_{14}$ & 8.0 & 8.5 \\
$\mathrm{n}-\mathrm{C}_{6} \mathrm{~F}_{14}$ & 8.5 & 9.3 \\
$\mathrm{n}-\mathrm{C}_{7} \mathrm{~F}_{16}$ & 7.2 & 8.2 \\
\hline
\end{tabular}




\section{Global Emission Rates}

Table S2. Global annual emission rates $\left(\mathrm{Gg} \mathrm{yr}^{-1}\right)$ for all six PFCs used in the model simulations to obtain the best fit of the simulated model mixing ratios to the measured mixing ratios in Cape Grim.

\begin{tabular}{|c|c|c|c|c|c|c|}
\hline Year & c- $\mathrm{C}_{4} \mathrm{~F}_{8}$ & $\mathrm{n}-\mathrm{C}_{4} \mathrm{~F}_{10}$ & $\mathrm{n}-\mathrm{C}_{5} \mathrm{~F}_{12}$ & i- $\mathrm{C}_{6} \mathrm{~F}_{14}$ & $\mathrm{n}-\mathrm{C}_{6} \mathrm{~F}_{14}$ & $\mathrm{n}-\mathrm{C}_{7} \mathrm{~F}_{16}$ \\
\hline 1978 & 0.910 & 0.140 & 0.110 & 0.030 & 0.153 & 0.048 \\
\hline 1979 & 0.935 & 0.160 & 0.120 & 0.030 & 0.155 & 0.048 \\
\hline 1980 & 0.970 & 0.178 & 0.130 & 0.030 & 0.159 & 0.048 \\
\hline 1981 & 1.030 & 0.186 & 0.140 & 0.030 & 0.160 & 0.075 \\
\hline 1982 & 1.110 & 0.194 & 0.150 & 0.030 & 0.162 & 0.100 \\
\hline 1983 & 1.20 & 0.202 & 0.160 & 0.030 & 0.164 & 0.120 \\
\hline 1984 & 1.328 & 0.210 & 0.170 & 0.030 & 0.166 & 0.140 \\
\hline 1985 & 1.476 & 0.218 & 0.190 & 0.030 & 0.170 & 0.183 \\
\hline 1986 & 1.673 & 0.226 & 0.208 & 0.035 & 0.170 & 0.183 \\
\hline 1987 & 1.476 & 0.234 & 0.220 & 0.035 & 0.170 & 0.183 \\
\hline 1988 & 1.230 & 0.242 & 0.233 & 0.035 & 0.170 & 0.183 \\
\hline 1989 & 0.984 & 0.254 & 0.245 & 0.035 & 0.170 & 0.183 \\
\hline 1990 & 0.787 & 0.264 & 0.258 & 0.035 & 0.170 & 0.183 \\
\hline 1991 & 0.590 & 0.272 & 0.270 & 0.035 & 0.170 & 0.183 \\
\hline 1992 & 0.443 & 0.279 & 0.284 & 0.119 & 0.170 & 0.183 \\
\hline 1993 & 0.394 & 0.283 & 0.300 & 0.150 & 0.170 & 0.183 \\
\hline 1994 & 0.394 & 0.284 & 0.310 & 0.180 & 0.250 & 0.183 \\
\hline 1995 & 0.445 & 0.285 & 0.318 & 0.230 & 1.150 & 0.183 \\
\hline 1996 & 0.500 & 0.286 & 0.315 & 0.250 & 1.200 & 0.183 \\
\hline 1997 & 0.555 & 0.287 & 0.285 & 0.250 & 1.210 & 0.183 \\
\hline 1998 & 0.610 & 0.284 & 0.243 & 0.190 & 0.900 & 0.183 \\
\hline 1999 & 0.665 & 0.274 & 0.220 & 0.168 & 0.653 & 0.183 \\
\hline 2000 & 0.720 & 0.240 & 0.197 & 0.145 & 0.470 & 0.183 \\
\hline 2001 & 0.775 & 0.200 & 0.173 & 0.120 & 0.395 & 0.183 \\
\hline 2002 & 0.830 & 0.170 & 0.150 & 0.105 & 0.358 & 0.183 \\
\hline 2003 & 0.895 & 0.140 & 0.120 & 0.093 & 0.330 & 0.183 \\
\hline 2004 & 0.950 & 0.118 & 0.095 & 0.088 & 0.311 & 0.183 \\
\hline 2005 & 1.005 & 0.110 & 0.080 & 0.085 & 0.293 & 0.183 \\
\hline 2006 & 1.060 & 0.103 & 0.075 & 0.085 & 0.267 & 0.183 \\
\hline 2007 & 1.115 & 0.098 & 0.070 & 0.085 & 0.243 & 0.183 \\
\hline 2008 & 1.170 & 0.095 & 0.065 & 0.085 & 0.218 & 0.183 \\
\hline 2009 & 1.225 & 0.093 & 0.060 & 0.085 & 0.199 & 0.183 \\
\hline 2010 & 1.280 & 0.092 & 0.058 & 0.085 & 0.179 & 0.183 \\
\hline 2011 & 1.335 & 0.092 & 0.057 & 0.085 & 0.160 & 0.183 \\
\hline 2012 & 1.390 & 0.092 & 0.057 & 0.085 & 0.150 & 0.183 \\
\hline 2013 & 1.470 & 0.092 & 0.057 & 0.085 & 0.141 & 0.183 \\
\hline 2014 & 1.570 & 0.092 & 0.057 & 0.085 & 0.141 & 0.183 \\
\hline 2015 & 1.670 & 0.092 & 0.057 & 0.085 & 0.141 & 0.183 \\
\hline 2016 & 1.779 & 0.092 & 0.057 & 0.085 & 0.141 & 0.183 \\
\hline 2017 & 1.900 & 0.092 & 0.057 & 0.085 & 0.141 & 0.183 \\
\hline
\end{tabular}


5 Correlations of PFC Mixing Ratios in Taiwan

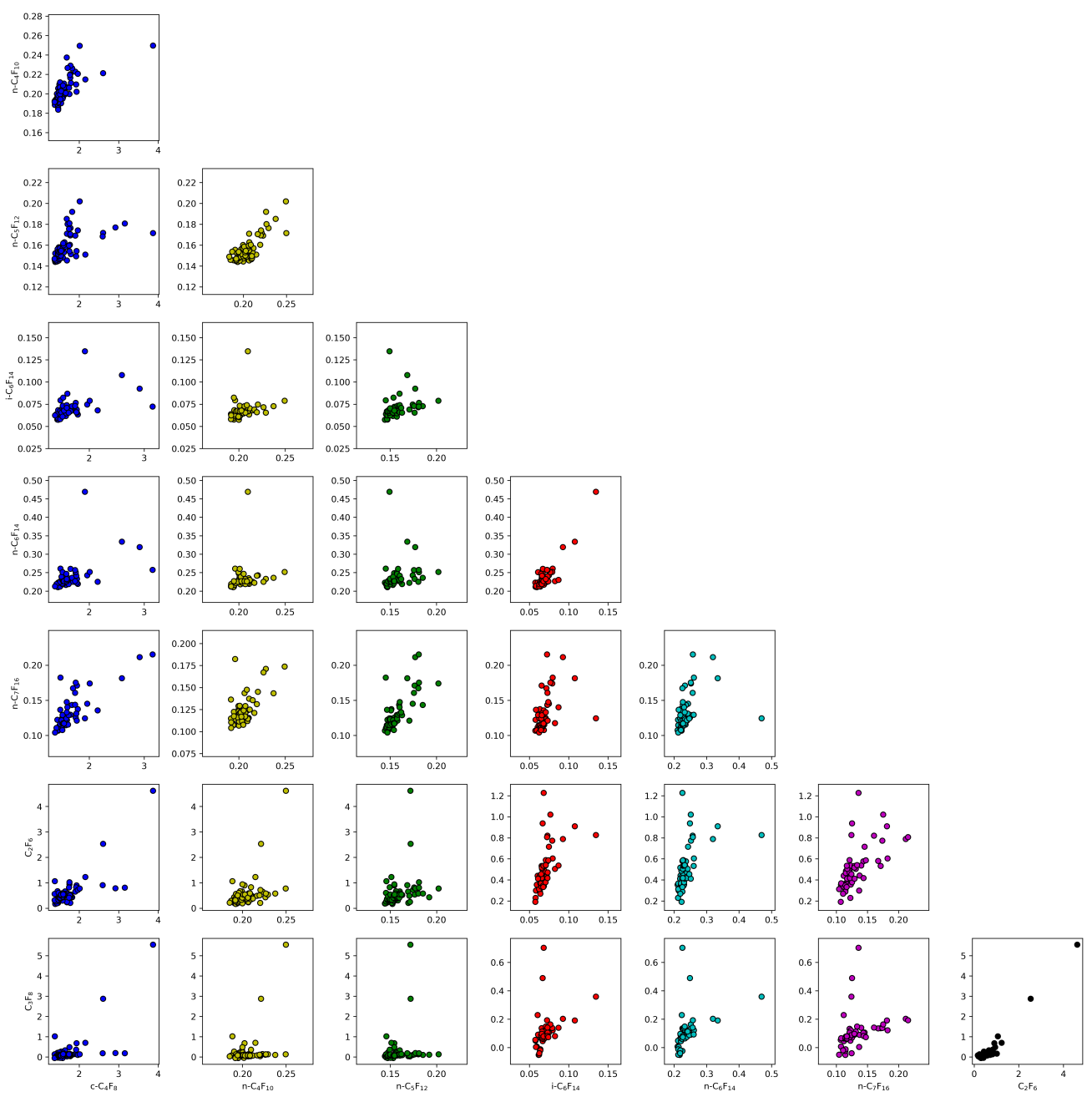

Figure S3. Correlations of all PFC mixing ratios (ppt) measured in Taiwan. 
Table S3. R-squared Spearman correlation coefficients for correlation analysis between all PFCs in this study. All values are significant (p-value $<0.05)$.

\begin{tabular}{|c|c|c|c|c|c|c|c|}
\hline & c- $-\mathrm{C}_{4} \mathrm{~F}_{8}$ & $\mathrm{n}-\mathrm{C}_{4} \mathrm{~F}_{10}$ & $\mathrm{n}-\mathrm{C}_{5} \mathrm{~F}_{12}$ & $\mathrm{i}-\mathrm{C}_{6} \mathrm{~F}_{14}$ & $\mathrm{n}-\mathrm{C}_{6} \mathrm{~F}_{14}$ & $\mathrm{n}-\mathrm{C}_{7} \mathrm{~F}_{16}$ & $\mathrm{C}_{2} \mathrm{~F}_{6}$ \\
\hline n- $C_{4} \mathrm{~F}_{10}$ & 0.62 & & & & & & \\
\hline $\mathrm{n}-\mathrm{C}_{5} \mathrm{~F}_{12}$ & 0.56 & 0.45 & & & & & \\
\hline $\mathrm{i}-\mathrm{C}_{6} \mathrm{~F}_{14}$ & 0.35 & 0.20 & 0.32 & & & & \\
\hline $\mathrm{n}-\mathrm{C}_{6} \mathrm{~F}_{14}$ & 0.35 & 0.20 & 0.33 & 0.40 & & & \\
\hline $\mathrm{n}-\mathrm{C}_{7} \mathrm{~F}_{16}$ & 0.55 & 0.23 & 0.49 & 0.33 & 0.39 & & \\
\hline $\mathrm{C}_{2} \mathrm{~F}_{6}$ & 0.42 & 0.21 & 0.28 & 0.52 & 0.52 & 0.54 & \\
\hline $\mathrm{C}_{3} \mathrm{~F}_{8}$ & 0.35 & 0.14 & 0.20 & 0.39 & 0.50 & 0.46 & 0.68 \\
\hline
\end{tabular}

Table S4. R-squared Spearman correlation coefficients for correlation analysis between all PFCs in this study and the particle density per region derived from NAME model results. Significance is indicated by * (p-value $<0.05)$.

\begin{tabular}{lllllllll}
\hline & $\mathrm{c}-\mathrm{C}_{4} \mathrm{~F}_{8}$ & $\mathrm{n}-\mathrm{C}_{4} \mathrm{~F}_{10}$ & $\mathrm{n}-\mathrm{C}_{5} \mathrm{~F}_{12}$ & $\mathrm{i}-\mathrm{C}_{6} \mathrm{~F}_{14}$ & $\mathrm{n}-\mathrm{C}_{6} \mathrm{~F}_{14}$ & $\mathrm{n}-\mathrm{C}_{7} \mathrm{~F}_{16}$ & $\mathrm{C}_{2} \mathrm{~F}_{6}$ & $\mathrm{C}_{3} \mathrm{~F}_{8}$ \\
\hline East China & $0.33^{*}$ & $0.20^{*}$ & $0.47^{*}$ & $0.33^{*}$ & $0.19^{*}$ & $0.49^{*}$ & $0.19^{*}$ & $0.10^{*}$ \\
North China & $0.02^{*}$ & 0.03 & $0.10^{*}$ & 0.02 & 0.01 & $0.07^{*}$ & 0.00 & 0.00 \\
North -East China & 0.02 & 0.01 & 0.01 & 0.02 & 0.01 & 0.00 & 0.01 & 0.00 \\
North-West China & 0.06 & 0.00 & 0.00 & 0.06 & 0.01 & 0.01 & 0.00 & 0.03 \\
South-Central China & $0.14^{*}$ & $0.09^{*}$ & $0.21^{*}$ & $0.14^{*}$ & 0.07 & $0.11^{*}$ & $0.13^{*}$ & 0.05 \\
South-West China & 0.01 & 0.00 & 0.00 & 0.01 & 0.00 & 0.02 & 0.02 & 0.02 \\
Indo-China & 0.00 & 0.01 & 0.00 & 0.00 & 0.00 & 0.01 & 0.03 & $0.05^{*}$ \\
Philippines & 0.03 & 0.01 & 0.01 & 0.03 & 0.05 & 0.01 & 0.04 & 0.01 \\
Taiwan & 0.00 & 0.00 & 0.03 & 0.00 & 0.02 & 0.02 & 0.02 & 0.04 \\
Japan & 0.01 & 0.01 & 0.01 & 0.01 & 0.01 & 0.00 & 0.00 & 0.01 \\
Korea & $0.08^{*}$ & 0.00 & 0.01 & $0.08^{*}$ & $0.14^{*}$ & 0.06 & $0.07^{*}$ & 0.01 \\
East China Sea & $0.19^{*}$ & $0.12^{*}$ & $0.33^{*}$ & $0.19^{*}$ & $0.27^{*}$ & $0.21^{*}$ & $0.12^{*}$ & $0.07^{*}$ \\
Japan Sea & 0.05 & 0.00 & 0.00 & 0.05 & 0.06 & 0.02 & 0.03 & 0.00 \\
Pacific Ocean & 0.03 & 0.01 & $0.06^{*}$ & 0.03 & 0.02 & 0.05 & 0.00 & 0.01 \\
South China Sea & 0.03 & 0.00 & 0.00 & 0.03 & 0.00 & 0.00 & $0.06^{*}$ & $0.05^{*}$ \\
\hline
\end{tabular}


Table S5. R-squared Spearman correlation coefficients for correlation analysis between all PFCs in this study and the CO mixing ratio derived from the NAME model results per CO source type. Significance is indicated by ${ }^{*}$ (p-value $<0.05$ ). Industry includes combustion and processing; power plants include energy generation, energy conversion, and extraction; waste includes landfills, waste water, and waste incineration; residential includes domestic and commercial residences; and agriculture includes animal husbandry, rice crops, and soil.

\begin{tabular}{lllllllll}
\hline & $\mathrm{c}-\mathrm{C}_{4} \mathrm{~F}_{8}$ & $\mathrm{n}-\mathrm{C}_{4} \mathrm{~F}_{10}$ & $\mathrm{n}-\mathrm{C}_{5} \mathrm{~F}_{12}$ & $\mathrm{i}-\mathrm{C}_{6} \mathrm{~F}_{14}$ & $\mathrm{n}-\mathrm{C}_{6} \mathrm{~F}_{14}$ & $\mathrm{n}_{-} \mathrm{C}_{7} \mathrm{~F}_{16}$ & $\mathrm{C}_{2} \mathrm{~F}_{6}$ & $\mathrm{C}_{3} \mathrm{~F}_{8}$ \\
\hline Industry & $0.45^{*}$ & $0.21^{*}$ & $0.48^{*}$ & $0.34^{*}$ & $0.25^{*}$ & $0.48^{*}$ & $0.28^{*}$ & $0.21^{*}$ \\
Power Plants & $0.41^{*}$ & $0.13^{*}$ & $0.27^{*}$ & $0.31^{*}$ & $0.26^{*}$ & $0.37^{*}$ & $0.34^{*}$ & $0.31^{*}$ \\
Solvents & $0.39^{*}$ & $0.21^{*}$ & $0.50^{*}$ & $0.30^{*}$ & $0.20^{*}$ & $0.44^{*}$ & $0.19^{*}$ & $0.12^{*}$ \\
Agricultural Waste Burning & $0.33^{*}$ & $0.18^{*}$ & $0.41^{*}$ & $0.26^{*}$ & $0.18^{*}$ & $0.41^{*}$ & $0.18^{*}$ & $0.08^{*}$ \\
Waste & 0.04 & 0.00 & 0.00 & 0.03 & $0.14^{*}$ & 0.04 & $0.06^{*}$ & 0.01 \\
Forest Burning & 0.00 & 0.00 & 0.01 & 0.01 & 0.02 & 0.00 & 0.00 & 0.01 \\
Grassland Burning & 0.01 & 0.00 & 0.01 & 0.01 & 0.01 & 0.02 & 0.00 & 0.00 \\
Residential & $0.42^{*}$ & $0.2^{*}$ & $0.45^{*}$ & $0.33^{*}$ & $0.22^{*}$ & $0.46^{*}$ & $0.25^{*}$ & $0.17^{*}$ \\
International Shipping & 0.01 & 0.00 & 0.00 & 0.00 & 0.02 & 0.00 & 0.04 & $0.05^{*}$ \\
Surface Transportation & $0.16^{*}$ & 0.02 & 0.03 & $0.10^{*}$ & $0.08^{*}$ & $0.08^{*}$ & $0.17^{*}$ & $0.19^{*}$ \\
Agriculture & 0.00 & 0.01 & 0.01 & 0.00 & 0.00 & 0.01 & 0.00 & 0.01 \\
\hline
\end{tabular}

